\title{
Management of Spot Blotch Disease of Wheat in Eastern Uttar Pradesh
}

\author{
Shivam Kumar*, Ghanshyam Verma, S.P. Singh, Krish Kumar and \\ Sandeep Vishwakarma \\ Department of Plant Pathology, Narendra Deva University of Agriculture \& Technology, \\ Kumarganj, Faizabad(U.P.)-224229, India \\ *Corresponding author
}

\section{A B S T R A C T}

\begin{tabular}{|l|}
\hline Ke y w o r d s \\
About wheat, Spot blotch, \\
$\begin{array}{l}\text { Pathogen, Disease, } \\
\text { Symptoms, Yield loss and } \\
\text { their management }\end{array}$ \\
\hline Article Info \\
\hline $\begin{array}{l}\text { Accepted: } \\
17 \text { July } 2018 \\
\text { Available Online: } \\
10 \text { August } 2018\end{array}$ \\
\hline
\end{tabular}

\section{Introduction}

Wheat (Triticum aestivum L.) is very important cereal crop after rice in India and major staple food of South Asian countries. Wheat crop in eastern Uttar Pradesh suffers from a number of fungal diseases. Spot blotch caused Bipolaris sorokinianais more severe than other diseases. It attacks at all the growth stages of the crop starting from the seedling to spikes, that's why it was thought desirable to work on management of spot blotch disease.

Wheat (Triticum aestivum L.) crop belongs to family Poaceae (Graminae). It is the most important cereal crop after rice in India and major staple food of South Asian region countries. Generally, wheat is a self-pollinated and hexaploid plant. Three species, namely Triticum astivum L. (Bread wheat), Triticum durum Desf. (Macroni or durum wheat), and Triticum dicoccum Schrank. (Emmer wheat), are commonly cultivated at present. India is the world's second largest wheat producer, behind china and ahead USA. It has revealed from the archaeological records that wheat was cultivated in Mohenjo-Daro and Harappa nearly 5000 year back (Pal, 1996). The important of wheat as a food of South Asia is well known. It is utilized for bread, cakes, cookies, noodles, pestri-products, chapatti \& morconi etc. Wheat is the staple cereal food, it is eaten in the form of chapattis. The stable cereal food wheat eaten in the form of 'puris' 
or in the form of 'upmo' (Cocked form suji or rawa). In addition to this wheat is also consumed in various other preparations such 'dalia', 'halwa', 'Sweet meals' etc. In most of the urban areas in the country the use of backed leavened bread, flakes, cakes, biscuits etc. is increasing at a fast rate. Beside staple food for human being, wheat straw is a good source of feed for a large population of cattle in our country. Wheat grain contains $60-68 \%$ starch, 8.0 to $15 \%$ protein, 1.5 to 2.0 fat, 2.0 2.5 cellulose and 1.5 to $2.0 \%$ minerals (Rathore, 2001).

Spot blotch or Helminthosporium leaf blight caused by Bipolaris sorokiniana (Sacc.) Shoem. is a most important disease of wheat in north eastern plains zone (NEPZ) representing warm and humid climate in India as well as in other South Asian countries. It is also increasing in North western plains zone (NWPZ) due to climatic change and causing losses in susceptible varieties (Singh, 2014). In India, foliar blight of wheat had been noticed as early as 1924 (Kulkarni, 1924), but it was not of much consequence till recently. In the recent past, with the change in cropping system, foliar blight has now become a major disease and wide in our country causing 2.72 to $36.24 \%$ yield losses under different agroclimatic zones (Parashar et al., 1995).

Bipolaris sorokiniana (Sacc.) Shoemaker is a seed and soil borne pathogen, causes head blight, seedling blight, foliar blight/ spot blotch, common root rot and black point of wheat, barley and other small cereal grains and grasses (Wiese, 1998). Symptoms mainly develop in the form of dark brown necrotic spots (boat shaped) occur on the coleoptiles, leaves, crowns, stems, and roots with or without yellow halo around these. Darkening of the sub crown internode is a characteristic symptom. Lesions on the leaves start as a few $\mathrm{mm}$ that extend as elongated dark brown spots greater than 1-2 cm (Chand et al., 2002). In the humid subtropics of South Asia, there is evidence of stress conditions, which favor foliar blight (Dubin and Bimb, 1994). SharmaPoudyal et al., (2005) has reported that seed treatment with Vitavax 200B and Carbendazine improves early plant establishment in heavy soil predominating areas where wheat is cultivated after rice.

\section{The pathogen}

The fungus spot blotch is caused by Bipolaris sorokiniana (Sacc.) Shoem. Syn. Drechslerasorokiniana (Sacc.) [Syn. Helminthosporium sativum, teleomorph Cochliobolus sativus] Subram and Jain, Cochliobolus sativus, Drechsleraex Dastur [anamorph Bipolaris sorokiniana (Sacc.) Shoem.] and several synonyms of the anamorph have been used like Helminthosporium sorokinianum, Drechslera sorokiniana and Helminthosporium sativum (Maraite et al., 1998). Bipolaris sorokinianais characterized by thick-walled, elliptical conidia (60-120 $\mu \mathrm{m} \times 12-20 \mu \mathrm{m})$ with 5-9 cells. In axenic culture, the mycelium is composed of hyphae interwoven as a loose cottony mass and appears as white or light to darkgrey depending on the isolates (Kumar et al., 2002).

\section{The disease}

Spot blotch caused by Bipolaris sorokiniana (Sacc. in Sorok.) Shoem. is an important disease of wheat. In India, foliar blight of wheat had been noticed as early as 1924 (Kulkarni, 1924), but it was not of much consequence till recently. It has been observed in several other countries (Dickson, 1956). Spot blotch caused by Helminthosporium sativum (Bipolaris sorokiniana) was wide spread in six states of India, namely Madhya Pradesh, Uttar Pradesh, Himanchal Pradesh, West Bengal, Punjab and Haryana (Neema and Joshi 1973). 


\section{Symptomatology}

Symptoms mainly develop on sub-crown internodes, stem, leaves, awns, glumes and seeds. The main symptom caused by the pathogen is spot blotch, which is nothing but the disease of leaves. The early lesions on leaves are 1-2 mm long, small and dark brown in colour. The dark brown necrotic spots (boat shaped) occur on the coleoptiles, leaves, crowns, stems, and roots with or without yellow halo around these. Darkening of the sub crown internode is a characteristic symptom.

\section{Management of spot blotch disease of wheat using Bio-agents and Fungicides}

Singh et al., (2017) results showed that the seed treatment with vitavax power @ $3 \mathrm{~g} \mathrm{~kg}$ of seed followed by two spray of propiconazole @ $0.1 \%$ at the time of disease initiation on flag -1 leaf and at soft dough stage were best and per cent disease intensity (39.03\%) was minimum. Yadav et al., (2015) reported the effect of recommended dose of fungicides (Propiconazole, Carbendazim and Hexaconazole), bio-agents and botanicals on incidence and severity of spot blotch disease and seed yield of wheat. Two sprays of Carbendazim at $0.1 \%$ at tillering and boot leaf stage resulted in the maximum reduction in spot blotch incidence and severity followed by two applications of Propiconazole at tillering and boot leaf stage.

Singh et al., (2017) reported effect of seed treatment and foliar spray with fungicides and $T$. viride on disease intensity of spot blotch and yield contributing characters like ear length, number of grains/ ear, thousand grain weight, yield and avoidable yield losses were studied. Yadav et al., (2015) reported Triazole group Propiconazole especially have proven to be very effective against spot blotch disease. Singh et al., (2017) showed that the seed treatment with vitavax power@3 g/ kg of seed followed by two sprays of propiconazole @ $0.1 \%$ at the time of disease initiation on flag -1 leaf and at soft dough stage were best in managing the spot blotch disease. Yadav et al., (2015) reported Triazole group Propiconazole especially have proven to be very effective against spot blotch disease. Singh et al., (2014) reported seed treatment with Vitavax Power@0.25\% and two sprays of Tilt @ $0.1 \%$, highest average grain yields (42.78 q/ha) and thousand grain weight were in case of in NEPZ, and $57.30 \mathrm{q} / \mathrm{ha}$ at Karnal (NWPZ) with gain of $25.7 \%$ and $10.6 \%$, respectively, over untreated.

Singh D.P. (2014) reported the disease appeared at flag leaf visible stage on lower leaves and moved upward in NEPZ whereas it appeared relatively late at boot swollen stage in NWPZ. The first spray was given at boot leaf stage in majority of cases and second after 15 days of it. The lowest spot blotch score (35) was in case of Vitavax power @ $2.5 \mathrm{~g} / \mathrm{kg}$ of seed and two sprays of propiconazole (Tilt) @ 0.1\%.Yadav et al., (2015) reported Triazole group Propiconazole especially have proven to be very effective against spot blotch disease.

This experiment was conducted at laboratory of Student instructional farm of Plant Pathology, Narendra Deva University of Agriculture and Technology. The experimental details are given below.

Treatments-13

Variety: Raj 4015

Plot size: $2 \times 2 \mathrm{~m}^{2}$

Design: RBD

Replications: 4

\section{Observations to be recorded}

Disease incidence and severity 1000 seed wt. (g.)

Yield (q/ha) 
Disease intensity was recorded on first appearance of symptoms after 07 days of $1^{\text {st }}$ and $2^{\text {nd }}$ spray. Observations on flag leaf and flag -1 leaf of 10 randomly selected plants from each treatment of each replication.

The per cent disease intensity (PDI) was calculated by using the formula:

\section{Per cent disease intensity}

It was calculated according to Mc Kinney (1975) formula

Sum of all disease rating Disease intensity $(\%)=$ X 100

Total number of rating $X$ maximum

Area under disease progress curve (Avalue)

It was calculated by the following formula (Dubin et al., 1998).

$$
\mathrm{AUDPC}=\Sigma_{\mathrm{i}=1}^{\mathrm{n}}[(\mathrm{Yi}+1+\mathrm{Yi}) \times 0.5][\mathrm{Ti}+1-\mathrm{Ti}]
$$

Where,

$\mathrm{Yi}=$ Severity/ intensity (\%) at the $\mathrm{i}^{\text {th }}$ observation.

Ti= Time (days) of $i^{\text {th }}$ observation

$\mathrm{n}=$ Total number of observation

First observation on disease intensity was recorded before first spraying using (Kumar et al., 1998). Subsequent observations were recorded before each spray and finally disease intensity was recorded 20 days after spray. Yield (q/ha), plant disease control and per cent increase in yield were also calculated.

Yield in treated plot - Yield in check plot Per cent increase in yield = ------------- $\mathrm{x} 100$ Yield in check plot
PDI in check-PDI in treatments Plant disease control (PDC) = ----------- X 100 PDI in check

Management of spot blotch disease of wheat using bio-agents and fungicides

For the management of spot blotch disease of wheat two bio-agent and four fungicides were used. Among these bio-agents such as Trichodermaviride@ $6 \mathrm{gm} / \mathrm{kg}$ seed $\left(\mathrm{T}_{3}\right)$ and Pseudomonas fluorescence@ $6 \mathrm{gm} / \mathrm{kg}$ seed $\left(\mathrm{T}_{4}\right)$ and fungicides vitavax 75 WP @ $2.5 \mathrm{gm}$ /kg, Thiram 75 WDP @ 3 gm/kg seed used as seed dressers. Whereas, propiconazole @ $0.1 \%$, first at boot leaf stage second after at 20 days as foliar spray and Nativo (trifloxysytrobin 25\% + tebuconazole 50\%) @ $0.4 \mathrm{gm} / \mathrm{lit}$, first at boot leaf stage second after at 20 days as foliar spray.

\section{Per cent disease intensity (PDI)}

The data on plant disease intensity (PDI) was recorded after second spray on wheat crop have been presented in table 16 clearly recoded that plant disease intensity decreased with the application of different treatment over untreated control. Minimum plant disease intensity was recorded (25.83per cent) under $\mathrm{T}_{5}$ (Seed treatment with vitavax 75 WP @ 2.5 $\mathrm{gm} / \mathrm{kg}$ seed +2 foliar sprays of propiconazole@0.1\%, first at boot leaf Stage second after 20 days). Which has highly significant with all the treatments. Hence, maximum plant disease intensity (PDI) was recorded under untreated control.

\section{AUDPC (Area under disease progressive curve)}

The data on AUDPC was recorded after second spray on wheat crop have been presented in table 16 clearly recoded that plant disease intensity decreased with the application of different treatment over untreated control. 
Table.1 Management of spot blotch disease of wheat using bio-agents and fungicides

\begin{tabular}{|c|c|}
\hline $\begin{array}{l}\text { S. } \\
\text { No. }\end{array}$ & Treatments \\
\hline $\mathbf{T}_{\mathbf{0}}$ & Untreated control (unsprayed) \\
\hline $\mathbf{T}_{1}$ & Seed treatment with vitavax 75 WP @ $2.5 \mathrm{gm} / \mathrm{kg}$ \\
\hline $\mathbf{T}_{2}$ & Seed treatment with Thiram 75 WDP @ 3 gm/kg \\
\hline $\mathbf{T}_{3}$ & Seed treatment with Trichoderma viride@6gm/kg of seed \\
\hline $\mathbf{T}_{4}$ & Seed treatment with Pseudomonas fluorescence@6gm/kg \\
\hline $\mathbf{T}_{5}$ & Seed treatment with vitavax 75 WP @ $2.5 \mathrm{gm} / \mathrm{kg}+2$ foliar sprays of propiconazole@ $0.1 \%$ (first at boot leaf Stage and second after 20 days) \\
\hline $\mathbf{T}_{6}$ & Seed treatment with Thiram 75 WDP @ 3 gm/kg + 2 foliar sprays of propiconazole@ 0.1\% (first at boot leaf Stage and second after 20 days) \\
\hline $\mathbf{T}_{7}$ & Seed treatment with Trichoderma viride @6gm/kg of seed + 2 foliar sprays of propiconazole@ 0.1\%(first at boot leaf Stage second after 20 days) \\
\hline $\mathbf{T}_{8}$ & Seed treatment with Pseudomonas fluorescence @ 6gm/kg + 2 foliar sprays of propiconazole@ 0.1\%(first at boot leaf Stage second after 20 days) \\
\hline $\mathbf{T}_{9}$ & $\begin{array}{l}\text { Seed treatment with vitavax } 75 \text { WP @ } 2.5 \mathrm{gm} / \mathrm{kg}+2 \text { foliar sprays of Nativo (trifloxysytrobin } 25 \%+\text { tebuconazole } 50 \% \text { )@ } 0.4 \text { gm/lit, (first at } \\
\text { boot leaf stage second after at } 20 \text { days) }\end{array}$ \\
\hline $\mathbf{T}_{10}$ & $\begin{array}{l}\text { Seed treatment with Thiram } 75 \text { WDP @ } 3 \text { gm/ } \mathrm{kg}+2 \text { foliar sprays of Nativo (trifloxysytrobin 25\% + tebuconazole 50\%) @0.4 gm/lit, (first at } \\
\text { boot leaf stage second after at } 20 \text { days) }\end{array}$ \\
\hline $\mathbf{T}_{11}$ & $\begin{array}{l}\text { Seed treatment with Trichoderma viride @ } 6 \mathrm{gm} / \mathrm{kg} \text { of seed }+2 \text { foliar sprays of Nativo (trifloxysytrobin } 25 \%+\text { tebuconazole } 50 \% \text { ) @ } 0.4 \text { gm/lit, } \\
\text { (first at boot leaf stage second after at } 20 \text { days) }\end{array}$ \\
\hline $\mathbf{T}_{12}$ & $\begin{array}{l}\text { Seed treatment with Pseudomonas fluorescence @ } 6 \mathrm{gm} / \mathrm{kg}+2 \text { foliar sprays of Nativo (trifloxysytrobin } 25 \%+\text { tebuconazole } 50 \% \text { ) @ } 0.4 \text { gm/lit, (first } \\
\text { at boot leaf stage second after at } 20 \text { days) }\end{array}$ \\
\hline
\end{tabular}


Table.2 Effect of per cent disease intensity and AUDPC of spot blotch disease of Wheat

\begin{tabular}{|c|c|c|c|c|c|}
\hline \multirow[t]{2}{*}{ S. No. } & \multirow[t]{2}{*}{ Treatments } & \multicolumn{3}{|c|}{ Plant disease intensity(PDI) } & \multirow[t]{2}{*}{ AUDPC } \\
\hline & & Before spray & $\begin{array}{c}\text { After First } \\
\text { spray }\end{array}$ & $\begin{array}{c}\text { After Second } \\
\text { spray }\end{array}$ & \\
\hline $\mathbf{T}_{\mathbf{0}}$ & Untreated control (unsprayed & $\begin{array}{c}15.56 \\
(23.19)\end{array}$ & $\begin{array}{c}57.50 \\
(49.31)\end{array}$ & $\begin{array}{c}78.61 \\
(62.44)\end{array}$ & 732.1 \\
\hline $\mathbf{T}_{1}$ & Seed treatment with vitavax 75 WP @ 2.5 gm $/ \mathrm{kg}$ & $\begin{array}{c}2.78 \\
(2.98)\end{array}$ & $\begin{array}{c}35.00 \\
(36.27)\end{array}$ & $\begin{array}{c}56.39 \\
(48.42)\end{array}$ & 452.1 \\
\hline $\mathbf{T}_{2}$ & Seed treatment with Thiram @ 3 gm $/ \mathrm{kg}$ & $\begin{array}{c}3.89 \\
(3.58)\end{array}$ & $\begin{array}{c}45.83 \\
(42.59)\end{array}$ & $\begin{array}{c}65.83 \\
(55.37)\end{array}$ & 564.9 \\
\hline $\mathbf{T}_{3}$ & Seed treatment with Trichoderma viride@4gm $/ \mathrm{kg}$ of seed & $\begin{array}{c}4.50 \\
(3.89)\end{array}$ & $\begin{array}{c}47.50 \\
(43.57)\end{array}$ & $\begin{array}{c}67.78 \\
(49.06)\end{array}$ & 585.5 \\
\hline $\mathbf{T}_{4}$ & Seed treatment withPseudomonas fluorescence@4gm/kg & $\begin{array}{c}3.33 \\
(3.26)\end{array}$ & $\begin{array}{c}39.72 \\
(39.06)\end{array}$ & $\begin{array}{c}58.06 \\
(30.53)\end{array}$ & 492.9 \\
\hline $\mathbf{T}_{5}$ & $\begin{array}{l}\text { Seed treatment with vitavax } 75 \text { WP @ } 2.5 \mathrm{gm} / \mathrm{kg}+2 \text { foliar sprays of } \\
\text { propiconazole@ } 0.1 \%\end{array}$ & $\begin{array}{l}1.39 \\
(2.07)\end{array}$ & $\begin{array}{c}20.28 \\
(26.71)\end{array}$ & $\begin{array}{c}25.83 \\
(30.53)\end{array}$ & 237.2 \\
\hline $\mathrm{T}_{6}$ & $\begin{array}{l}\text { Seed treatment with Thiram @ } 3 \mathrm{gm} / \mathrm{kg}+2 \text { foliar sprays of } \\
\text { propiconazole@ } 0.1 \%\end{array}$ & $\begin{array}{l}3.06 \\
(3.14)\end{array}$ & $\begin{array}{l}23.89 \\
(29.27)\end{array}$ & $\begin{array}{l}35.56 \\
(36.57)\end{array}$ & 302.4 \\
\hline $\mathbf{T}_{7}$ & $\begin{array}{l}\text { Seed treatment with Trichodermaviride @ } 4 \mathrm{gm} / \mathrm{kg} \text { of seed }+2 \text { foliar } \\
\text { sprays of propiconazole@ } 0.1 \%\end{array}$ & $\begin{array}{c}4.44 \\
(3.53)\end{array}$ & $\begin{array}{c}25.83 \\
(30.53)\end{array}$ & $\begin{array}{c}36.67 \\
(37.29)\end{array}$ & 324.7 \\
\hline $\mathbf{T}_{8}$ & $\begin{array}{l}\text { Seed treatment with Pseudomonas fluorescence@ } 9 \mathrm{gm} / \mathrm{kg}+2 \text { foliar } \\
\text { sprays of propiconazole@ } 0.1 \%\end{array}$ & $\begin{array}{c}3.61 \\
(3.61)\end{array}$ & $\begin{array}{c}25.83 \\
(30.53)\end{array}$ & $\begin{array}{c}35.56 \\
(36.57)\end{array}$ & 317.9 \\
\hline$T_{9}$ & $\begin{array}{l}\text { Seed treatment with vitavax } 75 \text { WP @ } 2.5 \mathrm{gm} / \mathrm{kg}+2 \text { foliar sprays of } \\
\text { Nativo (trifloxysytrobin } 25 \% \text { + tebuconazole } 50 \% \text { ) @ } 0.4 \mathrm{gm} / \mathrm{lit}\end{array}$ & $\begin{array}{c}2.22 \\
(2.62)\end{array}$ & $\begin{array}{c}26.39 \\
(30.83)\end{array}$ & $\begin{array}{c}45.83 \\
(42.59)\end{array}$ & 352.9 \\
\hline $\mathbf{T}_{10}$ & $\begin{array}{l}\text { Seed treatment with Thiram @ } 3 \mathrm{gm} / \mathrm{kg}+2 \text { foliar sprays of Nativo } \\
\text { (trifloxysytrobin } 25 \%+\text { tebuconazole } 50 \% \text { ) @ } 0.4 \mathrm{gm} / \mathrm{lit}\end{array}$ & $\begin{array}{c}3.89 \\
(3.53)\end{array}$ & $\begin{array}{l}36.67 \\
(37.29)\end{array}$ & $\begin{array}{c}47.50 \\
(43.57)\end{array}$ & 436.5 \\
\hline $\mathbf{T}_{11}$ & $\begin{array}{l}\text { Seed treatment with Trichodermaviride@ } @ \mathrm{gm} / \mathrm{kg} \text { of seed }+2 \text { foliar } \\
\text { sprays of Nativo (trifloxysytrobin } 25 \%+\text { tebuconazole } 50 \% \text { ) @ } 0.4 \mathrm{gm} / \mathrm{lit}\end{array}$ & $\begin{array}{c}4.72 \\
(3.92)\end{array}$ & $\begin{array}{c}45.56 \\
(42.48)\end{array}$ & $\begin{array}{c}57.22 \\
(49.14)\end{array}$ & 535.7 \\
\hline $\mathbf{T}_{12}$ & $\begin{array}{l}\text { Seed treatment with Pseudomonas fluorescence@ } 4 \mathrm{gm} / \mathrm{kg}+2 \text { foliar } \\
\text { sprays of Nativo (trifloxysytrobin } 25 \%+\text { tebuconazole } 50 \% \text { ) @ } 0.4 \mathrm{gm} / \mathrm{lit}\end{array}$ & $\begin{array}{c}4.44 \\
(3.53)\end{array}$ & $\begin{array}{c}40.83 \\
(39.70)\end{array}$ & $\begin{array}{c}56.67 \\
(48.72)\end{array}$ & 499.7 \\
\hline SEm \pm & & 0.44 & 3.07 & 0.58 & \\
\hline CD & & 1.27 & 9.15 & 1.67 & \\
\hline
\end{tabular}


Table.3 Evaluation of thousand seed weight (g.), seed yield q/ha and percent increase yield against spot blotch disease of wheat

\begin{tabular}{|c|c|c|c|}
\hline Treatments & $\begin{array}{l}\text { Thousand seed weight } \\
\text { (g.) }\end{array}$ & $\begin{array}{l}\text { Seed Yield } \\
\text { (q/ha) }\end{array}$ & $\begin{array}{l}\text { Per cent increase } \\
\text { yield }\end{array}$ \\
\hline $\mathbf{T}_{0}$ & $\begin{array}{c}36.19 \\
(36.94)\end{array}$ & $\begin{array}{c}31.28 \\
(33.95)\end{array}$ & 0.00 \\
\hline$\overline{T_{1}}$ & $\begin{array}{c}38.56 \\
(38.35)\end{array}$ & $\begin{array}{c}36.31 \\
(37.05)\end{array}$ & 16.08 \\
\hline$\overline{T_{2}}$ & $\begin{array}{c}37.62 \\
(37.82)\end{array}$ & $\begin{array}{c}35.38 \\
(36.45)\end{array}$ & 13.11 \\
\hline$\overline{T_{3}}$ & $\begin{array}{c}36.86 \\
(37.35)\end{array}$ & $\begin{array}{c}34.56 \\
(35.97)\end{array}$ & 10.49 \\
\hline$\overline{T_{4}}$ & $\begin{array}{c}36.21 \\
(36.99)\end{array}$ & $\begin{array}{c}33.44 \\
(35.30)\end{array}$ & 6.91 \\
\hline $\mathrm{T}_{5}$ & $\begin{array}{c}41.49 \\
(40.05)\end{array}$ & $\begin{array}{c}38.69 \\
(38.41)\end{array}$ & 23.69 \\
\hline $\mathrm{T}_{6}$ & $\begin{array}{c}39.66 \\
(39.00)\end{array}$ & $\begin{array}{c}37.12 \\
(37.58)\end{array}$ & 18.67 \\
\hline $\mathbf{T}_{7}$ & $\begin{array}{c}39.99 \\
(39.17)\end{array}$ & $\begin{array}{c}36.63 \\
(37.23)\end{array}$ & 17.10 \\
\hline$\overline{T_{8}}$ & $\begin{array}{c}39.22 \\
(38.76)\end{array}$ & $\begin{array}{c}36.25 \\
(36.99)\end{array}$ & 15.89 \\
\hline$\overline{T_{9}}$ & $\begin{array}{c}40.55 \\
(39.52)\end{array}$ & $\begin{array}{c}37.06 \\
(37.47)\end{array}$ & 18.48 \\
\hline $\mathbf{T}_{10}$ & $\begin{array}{c}39.04 \\
(38.65)\end{array}$ & $\begin{array}{c}36.44 \\
(37.11)\end{array}$ & 16.50 \\
\hline$\overline{T_{11}}$ & $\begin{array}{c}38.11 \\
(38.12)\end{array}$ & $\begin{array}{c}35.06 \\
(36.27)\end{array}$ & 12.08 \\
\hline$\overline{T_{12}}$ & $\begin{array}{c}38.75 \\
(38.47)\end{array}$ & $\begin{array}{c}35.56 \\
(36.57)\end{array}$ & 13.68 \\
\hline SEm \pm & 0.45 & 1.19 & \\
\hline CD & 1.28 & 3.63 & \\
\hline
\end{tabular}


Fig.1 (a) Conidia with conidiophore of $B$. sorokiniana of wheat (b) Conidia of B. sorokiniana and (c) Spot of B. sorokiniana of wheat
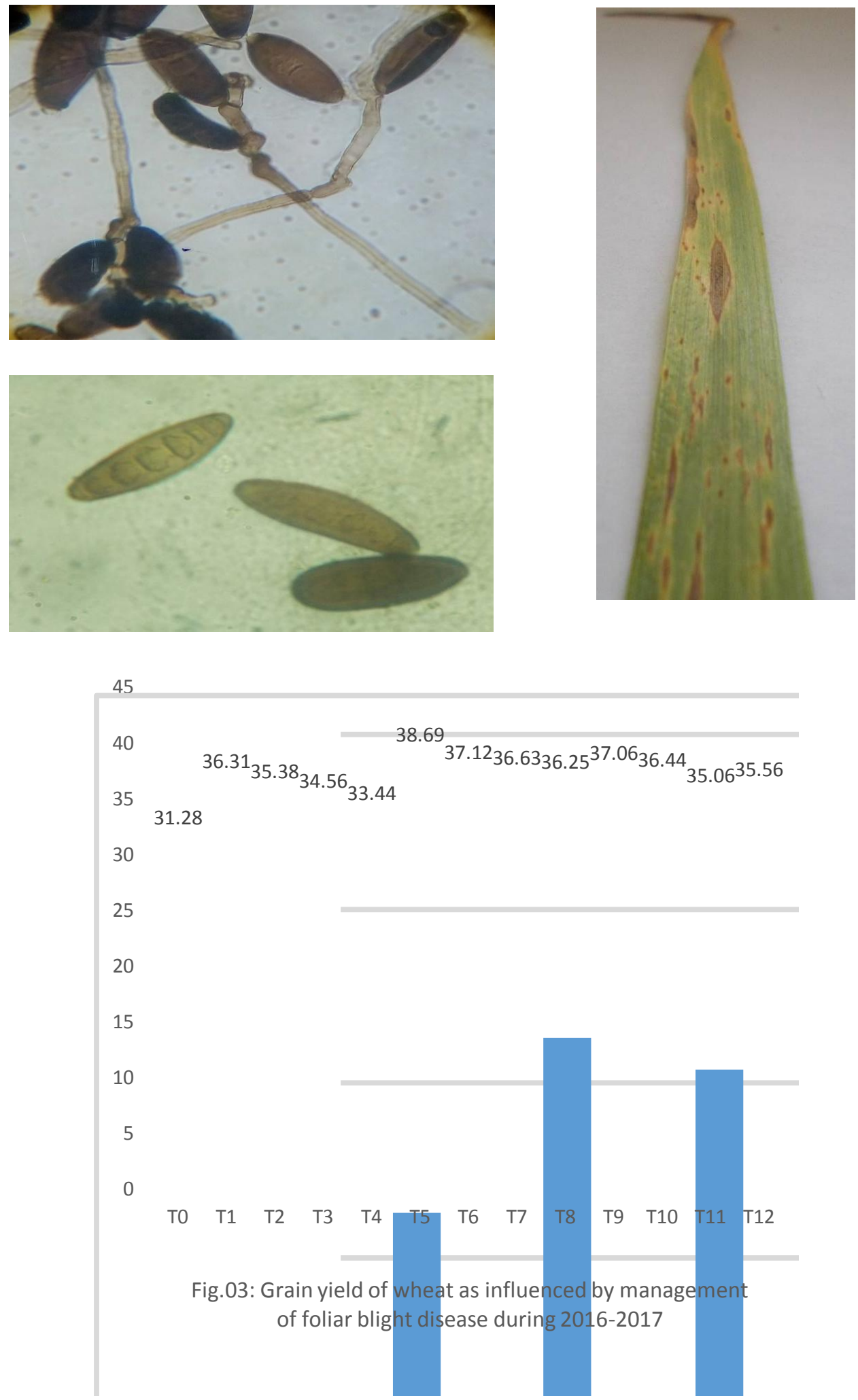


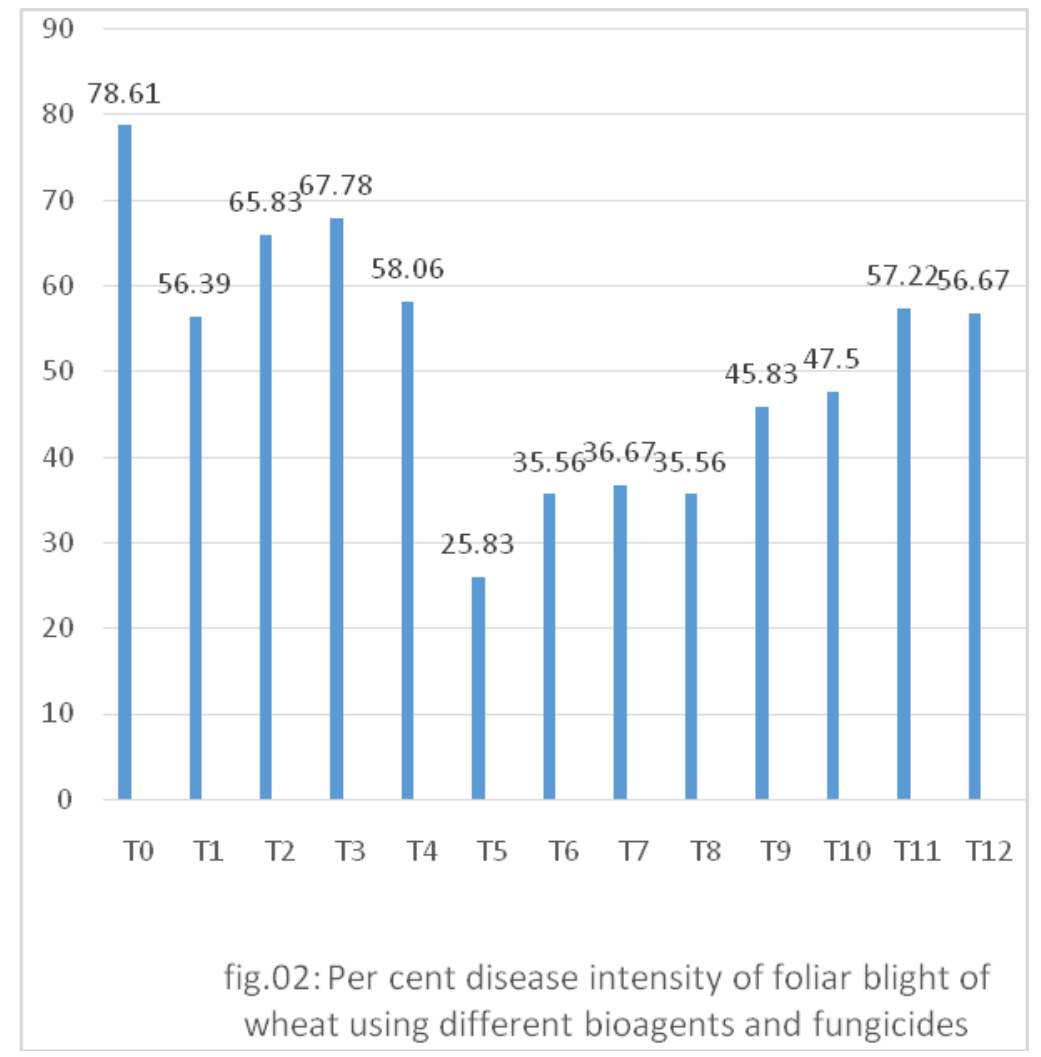

Maximum AUDPC was recorded (732.1) under $\mathrm{T}_{0} \quad$ (Untreated/control). Hence, Minimum AUDPC was recorded (237.2) under $\mathrm{T}_{5}$ (Seed treatment with vitavax $75 \mathrm{WP}$ @ $2.5 \mathrm{gm} / \mathrm{kg}$ seed +2 foliar sprays of propiconazole @ $0.1 \%$, first at boot leaf Stage second after 20 days). Which has highly significant with all the treatment.

\section{Thousand seed weight (g.)}

It is evident from table 17 that application of different fungicides has impact on Thousand seed weight in current study. Treatment $\mathrm{T}_{5}$ (Seed treatment with vitavax75 WP @2.5 $\mathrm{gm} / \mathrm{kg}+2$ foliar sprays of propiconazole@ $0.1 \%$ (first at boot leaf Stage and second after 20 days) and $\mathrm{T}_{9}$ (Seed treatment with vitavax 75 WP @ $2.5 \mathrm{gm} / \mathrm{kg}+2$ foliar sprays of Nativo (trifloxysytrobin 25\% + tebuconazole $50 \%) @ 0.4 \mathrm{gm} / \mathrm{lit}$, first at boot leaf stage second after at 20 days) were found significantly superior amongst all the treatment as highest seed weight i.e. $41.49 \mathrm{gm}$ and $40.55 \mathrm{gm}$, respectively. Were recorded with these treatment and treatment $T_{6}, T_{7}, T_{8}$ and $\mathrm{T}_{10}$ were second performer in cash of thousand seed weight which gave $39.66 \mathrm{gm}$, $39.99 \mathrm{gm}, 39.22 \mathrm{gm}, 39.04 \mathrm{gm}$ thousand seed weight, respectively. Least seed weight were recorded with treatment $\mathrm{T}_{0}$ (31.19 gm) which was at par with $\mathrm{T}_{3}\left(36.86 \mathrm{gm}\right.$.) and $\mathrm{T}_{4}(36.21$ gm).

\section{Seed yield (q/ha)}

Highest yield (38.69 q/ha) was recorded with $\mathrm{T}_{5}$ (Seed treatment with vitavax 75 WP @ 2.5 gm $/ \mathrm{kg}+2$ foliar sprays of propiconazole@ 0.1 per cent). This treatment was found significantly superior in cash ofyield $\mathrm{q} / \mathrm{ha}$ followed by $\mathrm{T}_{6}$ and $\mathrm{T}_{9}$ which gave the 37.12 $\mathrm{q} / \mathrm{ha}$ and $37.06 \mathrm{q} / \mathrm{ha}$, respectively.

\section{Per cent increase yield}

It is presented in table 17 clearly recoded that was maximum per cent increase yield (23.69) 
was recorded under $\mathrm{T}_{5}$ (Seed treatment with vitavax 75 WP @ $2.5 \mathrm{gm} / \mathrm{kg}$ seed + 2 foliar sprays of propiconazole @ $0.1 \%$, first at boot leaf Stage second after 20 days)followed by $\mathrm{T}_{6}(18.67)$ and $\mathrm{T}_{9}$ (18.48), respectively. However, Minimum was recorded (0.0) under $\mathrm{T}_{0}$ (control).

For the management of spot blotch disease of wheat two bio-agent and four fungicides were used.Plant disease intensity decreased with the application of different treatment over untreated control. Minimum plant disease intensity was recorded (25.83 per cent) under $\mathrm{T}_{5}$ (Seed treatment with vitavax 75 WP @ 2.5 $\mathrm{gm} / \mathrm{kg}$ seed +2 foliar sprays of propiconazole@0.1\%, first at boot leaf stage second after 20 days), Which has highly significant compared to all thetreatments. Maximum AUDPC was recorded (732.1) under $\mathrm{T}_{0}$ (Untreated/control). However, Minimum AUDPCwas recorded (237.2) under $\mathrm{T}_{5}$ (Seed treatment with vitavax $75 \mathrm{WP}$ @ $2.5 \mathrm{gm} / \mathrm{kg}$ seed +2 foliar sprays of propiconazole @ $0.1 \%$, first at boot leaf Stage second after 20 days).

All the fungicides gave significant impact on thousand seed weight over untreated control. Treatment $\mathrm{T}_{5}$ (Seed treatment with vitavax 75 WP @ $2.5 \mathrm{gm} / \mathrm{kg}+2$ foliar sprays of propiconazole@0.1\%, first at boot leaf Stage and second after 20 days) and $\mathrm{T}_{9}$ (Seed treatment with vitavax 75 WP @ $2.5 \mathrm{gm} / \mathrm{kg}+$ 2 foliar sprays of Nativo (trifloxysytrobin $25 \%$ + tebuconazole 50\%) @ 0.4 gm/lit, first at boot leaf stage second after at 20 days) were found significantly superior amongst all the treatment as thousand seed weight i.e. 41.49gm. and 40.55gm., respectively. Highest yield (38.69 q/ha) was recorded with $\mathrm{T}_{5}$ (Seed treatment with vitavax 75 WP @ $2.5 \mathrm{gm} / \mathrm{kg}+$ 2 foliar sprays of propiconazole@ 0.1per cent). This treatment was found significantly superior in cash ofyield $\mathrm{q} / \mathrm{ha}$ followed by $\mathrm{T}_{6}$ andT ${ }_{9}$ which gave the yield $37.12 \mathrm{q} / \mathrm{ha}$ and
$37.06 \mathrm{q} / \mathrm{ha}$, respectively. Maximum Per cent increase yield (23.69per cent) was recorded under $\mathrm{T}_{5}$ followed by $\mathrm{T}_{6}(18.67$ per cent) and $\mathrm{T}_{9}$ (18.48per cent), respectively. However, Minimum was recorded (0.0) under $\mathrm{T}_{0}$ (control). Seed treatment with Trichoderma viride and Pseudomonas fluorescence were not found effective alone because they have more disease intensity and lower yield compared the systemic fungicides such as propiconazole and Nativo (trifloxysytrobin $25 \%$ + tebuconazole 50\%), which were used as foliar spray with combination of seed treatment with vitavax $75 \mathrm{WP}$ and Thiram.

All the fungicides gave significant impact on thousand seed weight over untreated control. Treatment $\mathrm{T}_{5}$ (Seed treatment with vitavax 75 WP @ $2.5 \mathrm{gm} / \mathrm{kg}+2$ foliar sprays of propiconazole@0.1\%, first at boot leaf Stage and second after 20 days) and $\mathrm{T}_{9}$ (Seed treatment with vitavax 75 WP @ $2.5 \mathrm{gm} / \mathrm{kg}+$ 2 foliar sprays of Nativo (trifloxysytrobin $25 \%$ + tebuconazole 50\%) @ $0.4 \mathrm{gm} / \mathrm{lit}$, first at boot leaf stage second after at 20 days) were found significantly superior amongst all the treatment as thousand seed weight i.e. $41.49 \mathrm{gm}$ and $40.55 \mathrm{gm}$, respectively. Highest yield (38.69 q/ha) was recorded with $\mathrm{T}_{5}$ (Seed treatment with vitavax 75 WP @ $2.5 \mathrm{gm} / \mathrm{kg}+$ 2 foliar sprays of propiconazole@ 0.1per cent). This treatment was found significantly superior in cash of yield $\mathrm{q} /$ ha followed by $\mathrm{T}_{6}$ and $\mathrm{T}_{9}$ which gave the yield $37.12 \mathrm{q} / \mathrm{ha}$ and $37.06 \mathrm{q} / \mathrm{ha}$, respectively. Maximum Per cent increase yield (23.69per cent) was recorded under $\mathrm{T}_{5}$ followed by $\mathrm{T}_{6}(18.67$ per cent) and $\mathrm{T}_{9}$ (18.48per cent), respectively.

\section{References}

Anonymous (2016). Project Directorate report (IIW \& BR), Karnal.

Anonymous (2016). United states Department of Agriculture (USDA), Circular Series WAP, 8-16. 
Chand, R., Singh, H.V., Joshi, A.K. and Duveiller, E. (2002). Physiological and morphological aspects of Bipolaris sorokiniana conidia surviving on Wheat straw. Journalof Plant Pathology 18: 328-332.

Chand, R., Singh, H.V., Joshi, A.K. and Duveiller, E. (2002). Physiological and morphological aspects of Bipolaris sorokiniana conidia surviving on Wheat straw. Journal of Plant Pathology 18: 328-332.

Dickson, J.G. (1956). Diseases of field Crops. Tata Mc Graw Hill Publishing Co. Ltd. New Delhi. pp. 517.

Dubin, H.J. and Duveiller, E. (2000). Helminthosporium leaf blights of wheat: integrated control and prospects for the future. In: proc. Int. conf. integrated Plant Disease Management for Sustainable Agriculture., New Delhi, 10-15 Nov. 1997, vol. (1): 575-579.

Kulkarni, G.S. (1924). Report of the work done in plant pathology section during the year 1922-23. Ann. Rept. Deptt of Agric., Bombay Presidency: 167- 171.

Kumar, J., Schafer, P., Huckelhoven, R., Lungen, G., Baltruslhat, H., Stain, E., Nagarajan, S. and Kugel, K.H. (2002). Bipolaris sorokiniana, a cereal pathogen of global concern: cytological and molecular approaches towards better control. Molecular Plant Pathology, 3(4): 185-195.

Kumar, J., Singh, G. and Nagarajan, S. (1998). A field scale of leaf blight recording. Indian Wheat News Newsletter, 5(2): pp 3-4.

Maraite, H., Zinno, T.D.I., Longree, H., Daumerie, V. and Duveiller, E. (1998). Fungi association with foliar blight of wheat areas. In: Helminthosporium blights of wheat: Spot blotch and Tan spot (Duveiller E, Dubin HJ, Reeves J, McNab A, eds.) Mexico. D.F., Mexico: CIMMYT, 293-300.
Nema, K.G. and Johsi, L.M. (1973). Spot blotch disease of Wheat in relation to host temperature and moisture. Indian Phytopath., 26: 41-48.

Parashar, M., Nagarajan, S., Goel, L.B. and Kumar, J. (1995). Report of coordinated experiments 1994-95. Crop Protection, AICWIP. Directorate of Wheat Research, Karnal: 206.

Parashar, M., Nagarajan, S., Goel, L.B. and Kumar, J. (1995). Report of coordinated experiments 1994-95. Crop Protection, AICWIP. Directorate of Wheat Research, Karnal: 206.

Rathore, P.S. (2001). Techniques \& management of field crop production, $1^{\text {st }} \mathrm{ed}^{\mathrm{n}}$. Agrobios (India) Jodhpur, 96120.

Sharma-Poudyal D, Duveiller E, Sharma RC (2005) Effects of seed treatment and foliar fungicides on Helminthosporium Leaf Blight and performance of wheat in warmer growing conditions. $J$. Phytopatho. 153: 401-408.

Singh D.P., Kumar A., Solanki I. S., Singh S. P., Verma J., Mahapatra Sunita, Vaish S. S, Mukhopadhyay S.K. and. Dutta, S. (2014). Management of spot blotch of wheat caused by Bipolaris sorokiniana in wheat using fungicides. Directorate of Wheat Research, Karnal, Haryana, India, 1-3.

Singh, R., Sengar, R.M.S. and Singh, S. (2012). Incidence of foliar blight pathogens of wheat (Triticum aestivum L.) In Agra Region, Department of Botany, Indian J. L. Sci. 1(2): pp 39-41.

Singh, R.N. and Singh, A.K. (2006). Spot type Net blotch of barley caused by Pyrenophorateres $f$. spp. maculata in India and its management presented in National Symposium on "Biodiversity and Biotechnology: Research and Development needs in edible Mushrooms and crop disease 
management held at Department of Plant Pathology, G.B. Pant University of Agriculture \& Technology, Pantnagar-263145 on November, 9-11, pp. 16

Singh, R.V., Singh, A.K. and Singh, S.P. (1998). Distribution of pathogens causing foliar blight of Wheat in India and neighbouring countries In: Dueiller, E. Dubin, H.J., Reeves, J. and Mc Nab, (Eds.). Helminthosporium Blight of Wheat: Spot Blotch and Tan Spot pp. 59-62. Mexico, D.F. CIMMYT.
Wiese, M.V. (1998). Compendium of wheat diseases (3rd Ed). St. Paul, USA: APS Press, 112.

Wilson, V.E. and Murphy, H.C. (1964). Morphology and physiology of $H$. victoria and related species. Phytopathology 54: 147-150.

Yadav, B.; Singh, R. and Kumar, A. (2015). Management of spot blotch of wheat using Fungicides, Bio-agents and Botanicals, Department of Plant Pathology, Afri. J. Agril. Rese. 10(25), 2494-2500.

\section{How to cite this article:}

Shivam Kumar, Ghanshyam Verma, S.P. Singh, Krish Kumar and Sandeep Vishwakarma. 2018. Management of Spot Blotch Disease of Wheat in Eastern Uttar Pradesh. Int.J.Curr.Microbiol.App.Sci. 7(08): 3184-3195. doi: https://doi.org/10.20546/ijcmas.2018.708.341 